\title{
Chromium Carbonyl Nitrosyls: Comparison with Isoelectronic Manganese Carbonyl Derivatives
}

\author{
Hongyan Wang, ${ }^{\mathrm{a}, \mathrm{b} *}$ Yaoming Xie, ${ }^{\mathrm{b}}$ Jun D. Zhang, ${ }^{\mathrm{b}} \mathrm{R}$. Bruce King, ${ }^{\mathrm{b} *}$ and Henry F. \\ Schaefer III ${ }^{\mathrm{b}}$ \\ ${ }^{a}$ Atomic and Molecular Physics Institute, Sichuan University \\ Chengdu 610065, P. R. China \\ and \\ ${ }^{\mathrm{b}}$ Department of Chemistry and Center for Computational Chemistry \\ University of Georgia, Athens, Georgia
}

Supporting Information

Tables S1 to S22. Harmonic vibrational frequencies (in $\mathrm{cm}^{-1}$ ) and infrared intensities (in parentheses, in $\mathrm{km} / \mathrm{mol})$ for the $\mathrm{Cr}_{2}(\mathrm{NO})_{2}(\mathrm{CO})_{n}$ derivatives $(n=8,7,6,5)$.

Tables S23 and S24. Harmonic vibrational frequencies (in $\mathrm{cm}^{-1}$ ) and infrared intensities (in parentheses, in $\mathrm{km} / \mathrm{mol}$ ) for two isomers of $\mathrm{Cr}(\mathrm{NO})_{2}(\mathrm{CO})_{3}$.

Table S25. Harmonic vibrational frequencies (in $\mathrm{cm}^{-1}$ ) and infrared intensities (in parentheses, in $\mathrm{km} / \mathrm{mol}$ ) for $\mathrm{Cr}(\mathrm{NO})_{2}(\mathrm{CO})_{2}$

Table S26. Harmonic vibrational frequencies $\left(\right.$ in $\mathrm{cm}^{-1}$ ) and infrared intensities (in parentheses, in $\mathrm{km} / \mathrm{mol})$ for $\mathrm{Cr}(\mathrm{NO})(\mathrm{CO})_{4}$. 
Table S1. Harmonic vibrational frequencies $\left(\right.$ in $\mathrm{cm}^{-1}$ ) and infrared intensities (in parentheses, in $\mathrm{km} / \mathrm{mol}$ ) for the structure $\mathbf{I a}$ of $\mathrm{Cr}_{2}(\mathrm{CO})_{8}(\mathrm{NO})_{2}$ with $D_{4 d}$ symmetry

\begin{tabular}{cccccc}
\hline & B3LYP & BP86 & & B3LYP & BP86 \\
\hline $\mathrm{a}_{1}$ & $92(0)$ & $92(0)$ & $\mathrm{e}_{1}$ & $53(0)$ & $51(0)$ \\
& $134(0)$ & $135(0)$ & & $91(1)$ & $88(1)$ \\
& $377(0)$ & $391(0)$ & & $101(0)$ & $98(0)$ \\
& $541(0)$ & $528(0)$ & & $410(2)$ & $389(1)$ \\
& $706(0)$ & $682(0)$ & & $448(28)$ & $459(16)$ \\
& $1859(0)$ & $1810(0)$ & & $549(2)$ & $536(1)$ \\
$\mathrm{a}_{2}$ & $2158(0)$ & $2071(0)$ & & $667(183)$ & $654(155)$ \\
$\mathrm{b}_{1}$ & $353(0)$ & $356(0)$ & & $2083(2569)$ & $1997(2230)$ \\
& $43(0)$ & $46(0)$ & & & \\
$\mathrm{b}_{2}$ & $109(0)$ & $357(0)$ & & & \\
& $364(33)$ & $196(5)$ & & & \\
& $519(226)$ & $511(172)$ & & & \\
& $705(248)$ & $682(216)$ & & & \\
& $1849(2683)$ & $1802(1957)$ & & & \\
$\mathrm{e}_{3}$ & $2077(595)$ & $2007(379)$ & & & \\
& $62(0)$ & $60(0)$ & $\mathrm{e}_{2}$ & $69(0)$ & $67(0)$ \\
& $81(0)$ & $78(0)$ & & $92(0)$ & $89(0)$ \\
& $100(0)$ & $96(0)$ & & $371(0)$ & $381(0)$ \\
& $393(0)$ & $382(0)$ & & $433(0)$ & $434(0)$ \\
& $446(0)$ & $458(0)$ & & $520(0)$ & $507(0)$ \\
& $546(0)$ & $533(0)$ & & $2073(0)$ & $1990(0)$ \\
& $675(0)$ & $662(0)$ & & & \\
& $2049(0)$ & $1963(0)$ & & & \\
\hline
\end{tabular}


Table S2. Harmonic vibrational frequencies $\left(\right.$ in $\mathrm{cm}^{-1}$ ) and infrared intensities (in parentheses, in $\mathrm{km} / \mathrm{mol}$ ) for the structure $\mathbf{I b}$ of $\mathrm{Cr}_{2}(\mathrm{CO})_{8}(\mathrm{NO})_{2}$ with $C_{1}$ symmetry

\begin{tabular}{|c|c|c|c|c|c|}
\hline & B3LYP & BP86 & & B3LYP & BP86 \\
\hline \multirow[t]{30}{*}{$\mathrm{a}$} & $33(0)$ & $31(0)$ & $\mathrm{a}$ & $438(6)$ & $445(1)$ \\
\hline & $43(0)$ & $37(0)$ & & $443(38)$ & $447(1)$ \\
\hline & $50(0)$ & $45(0)$ & & $450(7)$ & $454(2)$ \\
\hline & $51(0)$ & $48(0)$ & & $459(0)$ & $456(23)$ \\
\hline & $55(0)$ & $49(0)$ & & $464(7)$ & $468(2)$ \\
\hline & 61(1) & $51(1)$ & & $465(1)$ & $474(0)$ \\
\hline & $63(0)$ & $59(0)$ & & $505(1)$ & $495(1)$ \\
\hline & $80(0)$ & $76(0)$ & & $506(3)$ & $496(3)$ \\
\hline & $81(0)$ & $77(0)$ & & $517(0)$ & $508(1)$ \\
\hline & $87(0)$ & 83(0) & & $525(9)$ & $511(4)$ \\
\hline & $88(0)$ & $84(0)$ & & $525(5)$ & $511(7)$ \\
\hline & $91(0)$ & $88(0)$ & & $529(1)$ & $519(0)$ \\
\hline & $93(1)$ & $88(0)$ & & $552(3)$ & $542(1)$ \\
\hline & $96(0)$ & $92(0)$ & & $555(21)$ & $545(10)$ \\
\hline & $97(1)$ & $93(0)$ & & $664(619)$ & $664(380)$ \\
\hline & $99(0)$ & $96(0)$ & & $678(93)$ & $666(91)$ \\
\hline & $100(0)$ & $96(0)$ & & 681(43) & $668(152)$ \\
\hline & $103(0)$ & $100(0)$ & & $688(21)$ & $674(16)$ \\
\hline & 111(3) & 107(3) & & 691(114) & $676(102)$ \\
\hline & $142(0)$ & $142(0)$ & & $699(20)$ & $690(7)$ \\
\hline & $350(0)$ & $351(0)$ & & 1823(216) & 1784(173) \\
\hline & $351(0)$ & $351(0)$ & & 1867(1461) & $1815(1146)$ \\
\hline & $365(41)$ & $370(9)$ & & $2048(318)$ & $1964(607)$ \\
\hline & $377(1)$ & $381(0)$ & & $2053(187)$ & $1966(205)$ \\
\hline & $378(6)$ & $391(1)$ & & $2059(37)$ & 1973(34) \\
\hline & $380(1)$ & $393(7)$ & & 2063(1417) & 1978(873) \\
\hline & $384(3)$ & $398(0)$ & & $2080(2453)$ & 1992(1926) \\
\hline & $388(3)$ & $398(0)$ & & 2084(867) & $1999(700)$ \\
\hline & $402(1)$ & $406(6)$ & & $2090(1300)$ & 2014(1159) \\
\hline & $410(5)$ & $412(9)$ & & 2152(206) & $2064(172)$ \\
\hline
\end{tabular}


Table S3. Harmonic vibrational frequencies (in $\mathrm{cm}^{-1}$ ) and infrared intensities (in parentheses, in $\mathrm{km} / \mathrm{mol})$ for the structure $\mathbf{I c}$ of $\mathrm{Cr}_{2}(\mathrm{CO})_{8}(\mathrm{NO})_{2}$ with $C_{2 v}$ symmetry

\begin{tabular}{|c|c|c|c|c|c|}
\hline & B3LYP & BP86 & & B3LYP & BP86 \\
\hline \multirow[t]{19}{*}{$a_{1}$} & $65(0)$ & $64(0)$ & $\mathrm{b}_{1}$ & $60(0)$ & $55(0)$ \\
\hline & $94(1)$ & $87(0)$ & & $88(0)$ & $85(0)$ \\
\hline & $99(0)$ & $91(1)$ & & $91(1)$ & $85(0)$ \\
\hline & $122(0)$ & $120(0)$ & & $111(0)$ & $103(0)$ \\
\hline & $134(0)$ & $131(0)$ & & $347(0)$ & $348(0)$ \\
\hline & $152(1)$ & $142(0)$ & & 392(0) & $390(0)$ \\
\hline & $379(1)$ & $389(1)$ & & $444(44)$ & $455(26)$ \\
\hline & $382(8)$ & $397(0)$ & & $509(5)$ & $498(5)$ \\
\hline & $408(6)$ & $412(8)$ & & $558(14)$ & $545(6)$ \\
\hline & $458(0)$ & $446(0)$ & & $689(130)$ & $676(119)$ \\
\hline & $467(1)$ & $475(0)$ & & $2084(2621)$ & $1994(2297)$ \\
\hline & $523(10)$ & $510(8)$ & $\mathrm{b}_{2}$ & $38(0)$ & $33(0)$ \\
\hline & $534(1)$ & $527(1)$ & & $51(1)$ & $39(0)$ \\
\hline & $675(129)$ & $665(116)$ & & $85(0)$ & $81(0)$ \\
\hline & $696(18)$ & $690(5)$ & & $102(0)$ & $99(0)$ \\
\hline & $1887(1700)$ & $1830(1372)$ & & $122(4)$ & $116(4)$ \\
\hline & $2056(71)$ & 1972(49) & & $370(39)$ & $382(13)$ \\
\hline & 2091(732) & $2006(612)$ & & $379(2)$ & $395(2)$ \\
\hline & $2153(218)$ & $2065(180)$ & & $404(1)$ & $409(5)$ \\
\hline \multirow[t]{12}{*}{$a_{2}$} & $18 i$ & $19 i$ & & $457(16)$ & $456(0)$ \\
\hline & $34(0)$ & $26(0)$ & & $470(3)$ & $472(3)$ \\
\hline & $79(0)$ & $74(0)$ & & $519(0)$ & $507(0)$ \\
\hline & $89(0)$ & $85(0)$ & & $542(1)$ & 531(1) \\
\hline & $101(0)$ & $96(0)$ & & $675(671)$ & $665(16)$ \\
\hline & $349(0)$ & $349(0)$ & & $676(9)$ & $676(523)$ \\
\hline & $385(0)$ & $384(0)$ & & $1811(25)$ & $1773(5)$ \\
\hline & $438(0)$ & $453(0)$ & & $2046(578)$ & 1963(784) \\
\hline & $508(0)$ & $498(0)$ & & $2060(970)$ & $1978(353)$ \\
\hline & $556(0)$ & 543(0) & & $2087(1728)$ & $2012(1417)$ \\
\hline & $689(0)$ & $677(0)$ & & & \\
\hline & $2049(0)$ & $1961(0)$ & & & \\
\hline
\end{tabular}


Table S4. Harmonic vibrational frequencies (in $\mathrm{cm}^{-1}$ ) and infrared intensities (in parentheses, in $\mathrm{km} / \mathrm{mol}$ ) for the structure $\mathbf{I d}$ of $\mathrm{Cr}_{2}(\mathrm{CO})_{8}(\mathrm{NO})_{2}$ with $C_{2 h}$ symmetry

\begin{tabular}{|c|c|c|c|c|c|}
\hline & B3LYP & BP86 & & B3LYP & BP86 \\
\hline \multirow[t]{17}{*}{$a_{g}$} & $77(0)$ & $75(0)$ & \multirow[t]{13}{*}{$b_{g}$} & $51(0)$ & $48(0)$ \\
\hline & $78(0)$ & $75(0)$ & & $66(0)$ & $62(0)$ \\
\hline & $98(0)$ & $92(0)$ & & $86(0)$ & $83(0)$ \\
\hline & $118(0)$ & $114(0)$ & & $94(0)$ & $91(2)$ \\
\hline & $222(0)$ & $218(0)$ & & $186(0)$ & $175(0)$ \\
\hline & $371(0)$ & $381(2)$ & & $321(0)$ & $325(0)$ \\
\hline & $385(0)$ & $390(0)$ & & $353(0)$ & $354(0)$ \\
\hline & $401(0)$ & $396(0)$ & & $424(0)$ & $440(0)$ \\
\hline & $428(0)$ & $451(0)$ & & $493(0)$ & $478(0)$ \\
\hline & $449(0)$ & $461(0)$ & & $496(0)$ & $490(0)$ \\
\hline & $527(0)$ & $518(0)$ & & $540(0)$ & $524(0)$ \\
\hline & $590(0)$ & $585(0)$ & & $677(0)$ & $670(0)$ \\
\hline & $654(0)$ & $652(0)$ & & $2071(0)$ & 1981(466) \\
\hline & $1668(0)$ & $1623(0)$ & \multirow[t]{18}{*}{$\mathrm{b}_{\mathrm{u}}$} & $58(0)$ & $52(0)$ \\
\hline & $2036(0)$ & 1951(0) & & $64(2)$ & $61(1)$ \\
\hline & $2076(0)$ & 1987(0) & & $93(2)$ & $91(0)$ \\
\hline & $2151(0)$ & 2061(0) & & $98(1)$ & $91(0)$ \\
\hline \multirow[t]{14}{*}{$a_{u}$} & $26(0)$ & $24(0)$ & & $102(1)$ & $97(1)$ \\
\hline & $53(0)$ & $50(0)$ & & $244(35)$ & $247(20)$ \\
\hline & $80(0)$ & $77(0)$ & & $364(23)$ & $369(0)$ \\
\hline & $87(1)$ & $83(1)$ & & $382(3)$ & $390(0)$ \\
\hline & $358(7)$ & $365(3)$ & & $402(174)$ & $433(97)$ \\
\hline & $372(0)$ & $385(8)$ & & $419(77)$ & $436(45)$ \\
\hline & $426(7)$ & 441(4) & & $499(75)$ & $491(25)$ \\
\hline & $484(5)$ & $469(6)$ & & $516(6)$ & $500(5)$ \\
\hline & $523(0)$ & $511(0)$ & & $576(245)$ & $569(214)$ \\
\hline & $655(161)$ & $648(142)$ & & 640(999) & $643(873)$ \\
\hline & 1635(928) & $1599(750)$ & & $648(167)$ & $645(147)$ \\
\hline & $2077(1533)$ & $1987(1340)$ & & $2069(602)$ & $1980(0)$ \\
\hline & & & & $2072(2828)$ & $1983(2434)$ \\
\hline & & & & $2098(2618)$ & $2012(2435)$ \\
\hline
\end{tabular}


Table S5. Harmonic vibrational frequencies (in $\mathrm{cm}^{-1}$ ) and infrared intensities (in parentheses, in km/mol) for the structure IIa-s of $\mathrm{Cr}_{2}(\mathrm{CO})_{7}(\mathrm{NO})_{2}$ with $C_{s}$ symmetry

\begin{tabular}{|c|c|c|c|c|c|}
\hline & B3LYP & BP86 & & B3LYP & BP86 \\
\hline \multirow[t]{32}{*}{$\mathrm{a}^{\prime}$} & $35(0)$ & $31(0)$ & $\mathrm{a}^{\prime \prime}$ & $16(0)$ & $17(0)$ \\
\hline & $65(0)$ & $63(0)$ & & $20(0)$ & $20(0)$ \\
\hline & $73(1)$ & $73(2)$ & & $57(1)$ & $48(1)$ \\
\hline & $84(0)$ & $80(0)$ & & $67(0)$ & $65(0)$ \\
\hline & $91(0)$ & $90(1)$ & & $73(0)$ & $73(0)$ \\
\hline & $95(1)$ & $91(1)$ & & $85(0)$ & $85(0)$ \\
\hline & $99(1)$ & $94(1)$ & & $89(0)$ & $88(0)$ \\
\hline & $103(0)$ & $109(0)$ & & $99(1)$ & $96(0)$ \\
\hline & $112(2)$ & $118(2)$ & & $347(0)$ & $353(0)$ \\
\hline & $147(1)$ & $151(2)$ & & $356(0)$ & $358(0)$ \\
\hline & $365(30)$ & $366(0)$ & & $370(0)$ & $375(7)$ \\
\hline & $378(5)$ & $385(16)$ & & $373(0)$ & $384(0)$ \\
\hline & $399(4)$ & $396(0)$ & & $406(1)$ & $396(2)$ \\
\hline & $426(7)$ & $426(2)$ & & $429(0)$ & $434(5)$ \\
\hline & $426(7)$ & $426(2)$ & & $451(20)$ & $465(5)$ \\
\hline & $438(10)$ & $461(7)$ & & $457(11)$ & $471(5)$ \\
\hline & $453(12)$ & $469(8)$ & & $501(7)$ & $503(66)$ \\
\hline & $512(79)$ & $509(12)$ & & 541(1) & $522(1)$ \\
\hline & $523(8)$ & $510(8)$ & & $622(1)$ & $606(3)$ \\
\hline & $532(56)$ & $520(13)$ & & $673(116)$ & $654(100)$ \\
\hline & $549(2)$ & $532(18)$ & & $2043(122)$ & $1951(67)$ \\
\hline & $580(10)$ & $570(18)$ & & $2060(1771)$ & $1964(1407)$ \\
\hline & $655(117)$ & $637(63)$ & & $2063(525)$ & $1975(1200)$ \\
\hline & $677(34)$ & $657(50)$ & & & \\
\hline & 681(193) & $673(243)$ & & & \\
\hline & $703(103)$ & $687(10)$ & & & \\
\hline & $1835(800)$ & 1787(661) & & & \\
\hline & $1854(1396)$ & $1806(1082)$ & & & \\
\hline & $2050(399)$ & $1960(212)$ & & & \\
\hline & $2072(1396)$ & 1984(614) & & & \\
\hline & $2081(1287)$ & $1998(1031)$ & & & \\
\hline & 2139(198) & $2049(194)$ & & & \\
\hline
\end{tabular}


Table S6. Harmonic vibrational frequencies $\left(\right.$ in $\mathrm{cm}^{-1}$ ) and infrared intensities (in parentheses, in $\mathrm{km} / \mathrm{mol}$ ) for the structure IIb-s of $\mathrm{Cr}_{2}(\mathrm{CO})_{7}(\mathrm{NO})_{2}$ with $C_{1}$ symmetry

\begin{tabular}{|c|c|c|c|c|c|}
\hline & B3LYP & BP86 & & B3LYP & BP86 \\
\hline \multirow[t]{27}{*}{$\mathrm{a}$} & $11(0)$ & $24(0)$ & $\mathrm{a}$ & $436(10)$ & $434(3)$ \\
\hline & $19(0)$ & $34(0)$ & & $444(4)$ & $451(4)$ \\
\hline & $36(0)$ & 42(0) & & $450(12)$ & $462(12)$ \\
\hline & $64(0)$ & $55(0)$ & & $457(32)$ & $463(3)$ \\
\hline & $68(0)$ & 69(0) & & $477(2)$ & $469(15)$ \\
\hline & $72(0)$ & $72(0)$ & & $507(62)$ & $490(14)$ \\
\hline & $77(0)$ & $77(0)$ & & $515(17)$ & $502(8)$ \\
\hline & $82(1)$ & 81(0) & & $515(18)$ & $506(60)$ \\
\hline & $84(1)$ & $83(0)$ & & $529(8)$ & $518(9)$ \\
\hline & $86(0)$ & $84(0)$ & & $535(22)$ & $524(15)$ \\
\hline & $89(0)$ & $86(0)$ & & $537(39)$ & $532(15)$ \\
\hline & $93(1)$ & 89(1) & & $551(13)$ & $533(11)$ \\
\hline & $97(0)$ & 94(1) & & $630(30)$ & $617(43)$ \\
\hline & $100(0)$ & $97(0)$ & & $636(46)$ & $626(26)$ \\
\hline & $106(1)$ & $103(0)$ & & $678(74)$ & $647(66)$ \\
\hline & $113(0)$ & $121(1)$ & & $687(106)$ & $665(73)$ \\
\hline & $120(4)$ & $133(5)$ & & $689(242)$ & $671(219)$ \\
\hline & $149(2)$ & $160(1)$ & & 701(15) & $688(4)$ \\
\hline & $354(0)$ & $355(2)$ & & $1831(684)$ & $1790(536)$ \\
\hline & $359(5)$ & $360(1)$ & & 1847(1411) & 1797(1013) \\
\hline & $361(15)$ & $362(8)$ & & $2025(272)$ & 1936(233) \\
\hline & $376(8)$ & $372(5)$ & & $2044(596)$ & 1943(800) \\
\hline & $380(1)$ & $379(6)$ & & $2055(393)$ & $1955(276)$ \\
\hline & $382(0)$ & $385(1)$ & & $2065(550)$ & 1973(856) \\
\hline & $390(12)$ & $395(5)$ & & $2075(2207)$ & 1987(1221) \\
\hline & $396(8)$ & $398(5)$ & & 2089(1467) & 2004(1309) \\
\hline & $416(5)$ & $414(4)$ & & $2142(184)$ & $2048(236)$ \\
\hline
\end{tabular}


Table S7. Harmonic vibrational frequencies (in $\mathrm{cm}^{-1}$ ) and infrared intensities (in parentheses, in km/mol) for the structure IIc-s of $\mathrm{Cr}_{2}(\mathrm{CO})_{7}(\mathrm{NO})_{2}$ with $C_{2 v}$ symmetry

\begin{tabular}{|c|c|c|c|c|c|}
\hline & B3LYP & BP86 & & B3LYP & BP86 \\
\hline \multirow[t]{17}{*}{$a_{1}$} & $53(0)$ & $53(0)$ & $\mathrm{b}_{1}$ & $36 i$ & $51(0)$ \\
\hline & $78(0)$ & $76(0)$ & & $52(1)$ & $66(0)$ \\
\hline & $82(0)$ & $82(0)$ & & $68(0)$ & $83(2)$ \\
\hline & $91(1)$ & $90(0)$ & & $88(3)$ & $116 \mathrm{i}(0)$ \\
\hline & $222(1)$ & $227(0)$ & & $275(3)$ & $222(0)$ \\
\hline & $335(0)$ & $349(0)$ & & $341(1)$ & $341(2)$ \\
\hline & $367(0)$ & $372(0)$ & & $368(1)$ & $362(1)$ \\
\hline & $407(1)$ & $399(0)$ & & $419(0)$ & 413(0) \\
\hline & $454(9)$ & $470(0)$ & & $442(25)$ & $446(4)$ \\
\hline & $500(28)$ & $495(26)$ & & $473(21)$ & $469(21)$ \\
\hline & $559(10)$ & $548(4)$ & & $659(84)$ & $631(68)$ \\
\hline & $645(75)$ & $622(74)$ & & 1996(381) & $1917(403)$ \\
\hline & $695(2)$ & $680(4)$ & & $2065(2076)$ & 1973(1669) \\
\hline & $1848(1510)$ & $1795(1103)$ & $\mathrm{b}_{2}$ & $81(0)$ & $80(0)$ \\
\hline & $1896(219)$ & $1845(154)$ & & $97(0)$ & $95(0)$ \\
\hline & 2041(941) & 1944(757) & & $116(8)$ & $118(8)$ \\
\hline & $2133(251)$ & 2041(247) & & $195(0)$ & $145 i$ \\
\hline \multirow[t]{10}{*}{$a_{2}$} & $44(0)$ & $44(0)$ & & $203 i$ & 193(0) \\
\hline & $74(0)$ & $73(0)$ & & $264(38)$ & $266(40)$ \\
\hline & $109(0)$ & $110(0)$ & & $373(55)$ & $353(38)$ \\
\hline & $224 i$ & $219 i$ & & $445(2)$ & $466(0)$ \\
\hline & $242(0)$ & $241(0)$ & & $525(29)$ & $512(19)$ \\
\hline & $420(0)$ & $415(0)$ & & $543(115)$ & $528(120)$ \\
\hline & $427(0)$ & $436(0)$ & & $647(253)$ & $618(89)$ \\
\hline & $475(0)$ & $474(0)$ & & $652(197)$ & $640(270)$ \\
\hline & $611(0)$ & $595(0)$ & & $1836(1041)$ & $1786(795)$ \\
\hline & $2062(0)$ & $1971(0)$ & & $2080(2023)$ & 1994(1801) \\
\hline
\end{tabular}


Table S8. Harmonic vibrational frequencies (in $\mathrm{cm}^{-1}$ ) and infrared intensities (in parentheses, in $\mathrm{km} / \mathrm{mol}$ ) for the structure IId-t of $\mathrm{Cr}_{2}(\mathrm{CO})_{7}(\mathrm{NO})_{2}$ with $C_{1}$ symmetry

\begin{tabular}{|c|c|c|c|c|c|}
\hline & B3LYP & BP86 & & B3LYP & BP86 \\
\hline \multirow[t]{27}{*}{$\mathrm{a}$} & $18(0)$ & $13(0)$ & $\mathrm{a}$ & 392(1) & 409(20) \\
\hline & $25(0)$ & $22(0)$ & & $395(11)$ & $420(6)$ \\
\hline & $32(0)$ & $29(0)$ & & $405(17)$ & $430(10)$ \\
\hline & $42(0)$ & $40(0)$ & & $445(1)$ & $434(11)$ \\
\hline & $57(0)$ & $57(1)$ & & 451(10) & $448(4)$ \\
\hline & $61(1)$ & $58(0)$ & & $453(13)$ & $461(10)$ \\
\hline & $64(0)$ & $65(0)$ & & $463(13)$ & $472(4)$ \\
\hline & $72(1)$ & $71(0)$ & & $472(14)$ & $475(1)$ \\
\hline & $75(0)$ & $75(0)$ & & 494(54) & $489(37)$ \\
\hline & $83(0)$ & $81(0)$ & & $515(31)$ & $502(2)$ \\
\hline & $85(0)$ & $81(0)$ & & $518(5)$ & $508(10)$ \\
\hline & $86(1)$ & $83(0)$ & & $524(11)$ & $547(3)$ \\
\hline & $89(0)$ & $90(0)$ & & $542(14)$ & $589(193)$ \\
\hline & $98(1)$ & $94(0)$ & & $558(5)$ & $600(29)$ \\
\hline & $98(0)$ & $97(0)$ & & $596(11)$ & $610(21)$ \\
\hline & $102(1)$ & $102(0)$ & & $677(223)$ & $660(127)$ \\
\hline & 111(1) & $115(2)$ & & $680(96)$ & $666(117)$ \\
\hline & $146(3)$ & 149(1) & & $682(144)$ & $673(77)$ \\
\hline & $293(2)$ & $290(0)$ & & $1787(484)$ & $1755(344)$ \\
\hline & $306(37)$ & $334(4)$ & & $1816(1135)$ & $1780(977)$ \\
\hline & $322(0)$ & $343(10)$ & & $2024(250)$ & $1945(17)$ \\
\hline & $331(4)$ & $353(0)$ & & $2047(834)$ & $1965(294)$ \\
\hline & $343(6)$ & $357(0)$ & & $2051(654)$ & $1973(723)$ \\
\hline & $356(0)$ & $379(10)$ & & $2065(1682)$ & 1979(1211) \\
\hline & $374(15)$ & $388(3)$ & & $2101(1116)$ & 1986(1394) \\
\hline & $377(3)$ & $394(3)$ & & $2109(1387)$ & $1994(1448)$ \\
\hline & $384(4)$ & 402(2) & & $2145(177)$ & $2047(225)$ \\
\hline
\end{tabular}


Table S9. Harmonic vibrational frequencies (in $\mathrm{cm}^{-1}$ ) and infrared intensities (in parentheses, in $\mathrm{km} / \mathrm{mol}$ ) for the structure IIe-t of $\mathrm{Cr}_{2}(\mathrm{CO})_{7}(\mathrm{NO})_{2}$ with $C_{1}$ symmetry

\begin{tabular}{|c|c|c|c|c|c|}
\hline & B3LYP & BP86 & & B3LYP & BP86 \\
\hline \multirow[t]{27}{*}{$\mathrm{a}$} & $27(3)$ & $29(0)$ & $\mathrm{a}$ & $403(5)$ & $412(12)$ \\
\hline & $32(0)$ & $38(0)$ & & $417(17)$ & $421(11)$ \\
\hline & $38(0)$ & $44(0)$ & & $425(27)$ & $430(66)$ \\
\hline & $45(0)$ & $55(0)$ & & $435(11)$ & $438(4)$ \\
\hline & $65(2)$ & 64(1) & & $444(9)$ & $441(8)$ \\
\hline & $71(1)$ & $72(0)$ & & $454(10)$ & 451(29) \\
\hline & $74(0)$ & $74(0)$ & & $474(37)$ & $466(3)$ \\
\hline & $79(2)$ & $76(0)$ & & $501(21)$ & 492(14) \\
\hline & 81(1) & $79(1)$ & & $511(5)$ & $496(24)$ \\
\hline & $85(1)$ & $82(0)$ & & $518(20)$ & $502(5)$ \\
\hline & $86(2)$ & $86(0)$ & & $528(6)$ & $511(21)$ \\
\hline & $87(1)$ & $89(0)$ & & $541(14)$ & $523(18)$ \\
\hline & $91(1)$ & $90(1)$ & & $593(200)$ & $590(132)$ \\
\hline & $100(5)$ & $99(0)$ & & $614(30)$ & $605(44)$ \\
\hline & 101(8) & $114(0)$ & & $632(88)$ & $614(57)$ \\
\hline & $106(0)$ & $119(0)$ & & $639(69)$ & $625(43)$ \\
\hline & $129(12)$ & $183(7)$ & & $647(15)$ & $636(183)$ \\
\hline & $160(0)$ & 194(0) & & $654(64)$ & $662(8)$ \\
\hline & $209(7)$ & $260(7)$ & & 1827(776) & $1790(661)$ \\
\hline & $325(0)$ & $296(2)$ & & $1846(1414)$ & $1800(1026)$ \\
\hline & $339(9)$ & $346(22)$ & & $1949(228)$ & $1883(755)$ \\
\hline & $353(2)$ & $353(15)$ & & $2027(826)$ & $1906(471)$ \\
\hline & $355(1)$ & $361(2)$ & & $2038(681)$ & $1920(329)$ \\
\hline & $372(14)$ & $377(4)$ & & $2062(247)$ & $1973(372)$ \\
\hline & $375(2)$ & $389(1)$ & & 2068(2098) & 1982(1216) \\
\hline & $379(3)$ & $392(7)$ & & $2085(1735)$ & $1995(1731)$ \\
\hline & $384(1)$ & $397(2)$ & & $2134(202)$ & $2038(302)$ \\
\hline
\end{tabular}


Table S10. Harmonic vibrational frequencies (in $\mathrm{cm}^{-1}$ ) and infrared intensities (in parentheses, in $\mathrm{km} / \mathrm{mol}$ ) for the structure IIIa-s of $\mathrm{Cr}_{2}(\mathrm{CO})_{6}(\mathrm{NO})_{2}$ with $C_{2}$ symmetry

\begin{tabular}{|c|c|c|c|c|c|}
\hline & B3LYP & BP86 & & B3LYP & BP86 \\
\hline \multirow[t]{25}{*}{$\mathrm{a}$} & $13(0)$ & $12(0)$ & $\mathrm{b}$ & $45 i$ & $13(0)$ \\
\hline & $37(0)$ & $40(0)$ & & $45(0)$ & $63(0)$ \\
\hline & $74(0)$ & $72(0)$ & & $73(1)$ & $74(1)$ \\
\hline & $86(0)$ & $79(0)$ & & $89(1)$ & $85(1)$ \\
\hline & $89(0)$ & $89(1)$ & & $102(1)$ & $99(1)$ \\
\hline & $91(0)$ & $92(0)$ & & $115(0)$ & $112(0)$ \\
\hline & $127(0)$ & $127(0)$ & & $164(0)$ & $207(2)$ \\
\hline & $207(0)$ & $212(1)$ & & $253(0)$ & $290(4)$ \\
\hline & $217(0)$ & $223(0)$ & & $363(3)$ & $356(0)$ \\
\hline & $299(0)$ & $286(0)$ & & $379(1)$ & $373(0)$ \\
\hline & $357(1)$ & $366(0)$ & & $404(0)$ & $394(2)$ \\
\hline & $378(2)$ & $377(0)$ & & $418(24)$ & $435(6)$ \\
\hline & $398(1)$ & $393(2)$ & & $427(16)$ & $439(7)$ \\
\hline & $420(4)$ & $432(0)$ & & 473(9) & $474(2)$ \\
\hline & $451(2)$ & $462(1)$ & & $492(54)$ & $506(30)$ \\
\hline & $478(5)$ & $479(2)$ & & $554(27)$ & $538(47)$ \\
\hline & $528(21)$ & $511(14)$ & & $614(264)$ & $608(166)$ \\
\hline & $556(1)$ & $546(2)$ & & $634(114)$ & $623(104)$ \\
\hline & $617(1)$ & $605(0)$ & & $694(12)$ & $675(5)$ \\
\hline & $631(13)$ & $626(5)$ & & $1827(417)$ & $1776(138)$ \\
\hline & $701(39)$ & $680(36)$ & & 1968(972) & 1861(689) \\
\hline & $1856(1878)$ & $1803(1535)$ & & $2072(1463)$ & 1982(1281) \\
\hline & $1964(18)$ & $1857(0)$ & & 2091(2536) & $2002(2193)$ \\
\hline & $2072(351)$ & $1979(58)$ & & & \\
\hline & $2129(142)$ & $2034(185)$ & & & \\
\hline
\end{tabular}


Table S11. Harmonic vibrational frequencies (in $\mathrm{cm}^{-1}$ ) and infrared intensities (in parentheses, in $\mathrm{km} / \mathrm{mol}$ ) for the structure IIIb-s of $\mathrm{Cr}_{2}(\mathrm{CO})_{6}(\mathrm{NO})_{2}$ with $C_{2 v}$ symmetry

\begin{tabular}{|c|c|c|c|c|c|}
\hline & B3LYP & BP86 & & B3LYP & BP86 \\
\hline \multirow[t]{15}{*}{$a_{1}$} & $40(0)$ & $41(0)$ & $\mathrm{b}_{1}$ & $24 i$ & $16(0)$ \\
\hline & $82(0)$ & $80(0)$ & & $80(1)$ & $78(1)$ \\
\hline & $92(1)$ & $90(1)$ & & $89(2)$ & $86(1)$ \\
\hline & $96(0)$ & $94(0)$ & & $338(0)$ & $349(0)$ \\
\hline & $230(1)$ & $230(0)$ & & $378(4)$ & $373(0)$ \\
\hline & $361(0)$ & $370(0)$ & & $390(1)$ & $392(2)$ \\
\hline & 401(3) & $395(2)$ & & $424(16)$ & $439(10)$ \\
\hline & $453(1)$ & $467(0)$ & & $518(7)$ & $516(4)$ \\
\hline & $503(26)$ & $501(15)$ & & $628(67)$ & $618(49)$ \\
\hline & $564(3)$ & $551(3)$ & & 1948(927) & 1856(684) \\
\hline & $636(5)$ & $629(4)$ & & $2075(1587)$ & $1982(1325)$ \\
\hline & $703(53)$ & $679(38)$ & $\mathrm{b}_{2}$ & $48(0)$ & $62(0)$ \\
\hline & $1864(2032)$ & $1804(1558)$ & & $4(1)$ & $100(1)$ \\
\hline & $1950(1)$ & $1854(1)$ & & $116(0)$ & $113(0)$ \\
\hline & $2126(205)$ & 2033(203) & & $181(0)$ & 208(4) \\
\hline \multirow[t]{10}{*}{$a_{2}$} & $12 i$ & $10 i$ & & $279(0)$ & 292(7) \\
\hline & $66(0)$ & $56(0)$ & & $428(26)$ & $437(2)$ \\
\hline & $134(0)$ & $123(0)$ & & $464(0)$ & $474(0)$ \\
\hline & $189(0)$ & $178(0)$ & & $555(75)$ & $536(80)$ \\
\hline & $278(0)$ & $270(0)$ & & $620(250)$ & $612(209)$ \\
\hline & $373(0)$ & $370(0)$ & & $700(10)$ & $675(4)$ \\
\hline & 421(0) & $433(0)$ & & $1822(164)$ & 1775(107) \\
\hline & 491(0) & $480(0)$ & & $2089(2631)$ & $2002(2169)$ \\
\hline & $605(0)$ & $599(0)$ & & & \\
\hline & $2072(0)$ & $1979(0)$ & & & \\
\hline
\end{tabular}


Table S12. Harmonic vibrational frequencies (in $\mathrm{cm}^{-1}$ ) and infrared intensities (in parentheses, in $\mathrm{km} / \mathrm{mol}$ ) for the structure IIIc-s of $\mathrm{Cr}_{2}(\mathrm{CO})_{6}(\mathrm{NO})_{2}$ with $C_{2 v}$ symmetry

\begin{tabular}{|c|c|c|c|c|c|}
\hline & B3LYP & BP86 & & B3LYP & BP86 \\
\hline \multirow[t]{15}{*}{$a_{1}$} & $46(0)$ & $43(0)$ & $b_{1}$ & $60(0)$ & $57(0)$ \\
\hline & $81(0)$ & $79(0)$ & & $75(0)$ & $73(0)$ \\
\hline & 94(0) & $94(0)$ & & $82(0)$ & $79(0)$ \\
\hline & $113(2)$ & $108(1)$ & & $370(5)$ & $357(1)$ \\
\hline & $246(0)$ & $240(0)$ & & $373(3)$ & $382(1)$ \\
\hline & $387(4)$ & $388(2)$ & & 407(17) & $415(12)$ \\
\hline & $425(11)$ & $432(4)$ & & $426(2)$ & $432(1)$ \\
\hline & $456(15)$ & $462(5)$ & & $482(2)$ & $482(1)$ \\
\hline & $473(0)$ & $482(5)$ & & $613(56)$ & $608(53)$ \\
\hline & $545(2)$ & $531(0)$ & & $1624(952)$ & 1594(680) \\
\hline & $612(2)$ & $611(5)$ & & $2074(1210)$ & 1973(1048) \\
\hline & 680(77) & $665(59)$ & $\mathrm{b}_{2}$ & $67(0)$ & $67(0)$ \\
\hline & $1625(124)$ & 1598(76) & & $103(0)$ & $102(0)$ \\
\hline & $2069(1809)$ & 1973(1561) & & $106(0)$ & $105(0)$ \\
\hline & $2132(25)$ & 2034(29) & & 251(4) & $249(4)$ \\
\hline \multirow{10}{*}{$\mathrm{a}_{2}$} & $27(0)$ & $32(0)$ & & $368(3)$ & $383(5)$ \\
\hline & $87 i$ & $35 i$ & & 403(54) & $425(16)$ \\
\hline & $114(0)$ & $115(0)$ & & $440(1)$ & $467(0)$ \\
\hline & $178(0)$ & $229(0)$ & & $543(153)$ & $540(113)$ \\
\hline & $316(0)$ & $327(0)$ & & $575(59)$ & $575(117)$ \\
\hline & $356(0)$ & $361(0)$ & & $605(43)$ & $608(15)$ \\
\hline & $407(0)$ & $422(0)$ & & 2049(35) & 1957(3) \\
\hline & $522(0)$ & $520(0)$ & & 2091(2980) & $2000(2470)$ \\
\hline & $576(0)$ & $577(0)$ & & & \\
\hline & 2071(0) & 1971(0) & & & \\
\hline
\end{tabular}


Table S13. Harmonic vibrational frequencies (in $\mathrm{cm}^{-1}$ ) and infrared intensities (in parentheses, in km/mol) for the structure IIId-s of $\mathrm{Cr}_{2}(\mathrm{CO})_{6}(\mathrm{NO})_{2}$ with $C_{2 v}$ symmetry

\begin{tabular}{|c|c|c|c|c|c|}
\hline & B3LYP & BP86 & & $\bar{B} 3 \mathrm{LYP}$ & BP86 \\
\hline \multirow[t]{15}{*}{$\overline{a_{1}}$} & $71(0)$ & $70(1)$ & $\mathrm{b}_{1}$ & $18 i$ & $34 i$ \\
\hline & $80(0)$ & $79(0)$ & & $56(4)$ & $42(2)$ \\
\hline & $120(0)$ & $119(0)$ & & $82(0)$ & $82(0)$ \\
\hline & $136(0)$ & $126(0)$ & & $335(0)$ & $331(0)$ \\
\hline & $147(0)$ & $140(0)$ & & $364(0)$ & $371(2)$ \\
\hline & $379(11)$ & $387(4)$ & & $450(28)$ & $453(12)$ \\
\hline & 421(11) & $422(11)$ & & $497(14)$ & $501(12)$ \\
\hline & $450(0)$ & $482(0)$ & & $602(5)$ & $582(4)$ \\
\hline & $519(0)$ & $510(0)$ & & $2038(2018)$ & $1945(1666)$ \\
\hline & $586(7)$ & $578(2)$ & $\mathrm{b}_{2}$ & $35(0)$ & $13(0)$ \\
\hline & $654(60)$ & $646(61)$ & & $78(4)$ & $77(5)$ \\
\hline & $685(30)$ & $679(8)$ & & $97(0)$ & $95(0)$ \\
\hline & $1870(1692)$ & $1807(1328)$ & & $123(5)$ & $117(5)$ \\
\hline & $2067(605)$ & $1976(481)$ & & $371(35)$ & $380(15)$ \\
\hline & $2123(487)$ & 2029(419) & & $414(11)$ & $420(11)$ \\
\hline \multirow[t]{10}{*}{$a_{2}$} & $31 i$ & $33 i$ & & $445(19)$ & $477(1)$ \\
\hline & $56(0)$ & $45(0)$ & & $511(66)$ & $505(72)$ \\
\hline & $64 i$ & $84(0)$ & & $582(68)$ & $569(130)$ \\
\hline & $85(0)$ & $98 i$ & & $662(90)$ & $650(1)$ \\
\hline & $332(0)$ & $327(0)$ & & $672(337)$ & $674(277)$ \\
\hline & $351(0)$ & $350(0)$ & & $1790(8)$ & $1748(0)$ \\
\hline & $453(0)$ & $459(0)$ & & 2040(13) & 1953(18) \\
\hline & $498(0)$ & $506(0)$ & & $2071(2687)$ & $1987(2045)$ \\
\hline & $611(0)$ & $586(0)$ & & & \\
\hline & $2034(0)$ & $1941(0)$ & & & \\
\hline
\end{tabular}


Table S14. Harmonic vibrational frequencies (in $\mathrm{cm}^{-1}$ ) and infrared intensities (in parentheses, in $\mathrm{km} / \mathrm{mol}$ ) for the structure IIIe-t of $\mathrm{Cr}_{2}(\mathrm{CO})_{6}(\mathrm{NO})_{2}$ with $C_{2 v}$ symmetry

\begin{tabular}{|c|c|c|c|c|c|}
\hline & B3LYP & BP86 & & B3LYP & BP86 \\
\hline \multirow[t]{15}{*}{$a_{1}$} & $25(0)$ & $25(0)$ & $\mathrm{b}_{1}$ & $35(0)$ & $38(0)$ \\
\hline & $76(0)$ & $75(0)$ & & $72(0)$ & $70(1)$ \\
\hline & $85(0)$ & $83(0)$ & & $85(2)$ & $83(1)$ \\
\hline & $92(1)$ & $88(1)$ & & $350(1)$ & $350(0)$ \\
\hline & $204(0)$ & $204(0)$ & & $357(0)$ & $366(1)$ \\
\hline & $346(0)$ & $348(0)$ & & 391(7) & 401(1) \\
\hline & $382(1)$ & $378(1)$ & & $419(24)$ & $428(14)$ \\
\hline & $421(14)$ & $436(3)$ & & $440(2)$ & $435(4)$ \\
\hline & $488(38)$ & $479(32)$ & & $579(46)$ & $579(35)$ \\
\hline & $537(4)$ & $530(3)$ & & 1898(797) & $1823(585)$ \\
\hline & $602(26)$ & $610(18)$ & & $2073(1795)$ & 1981(1483) \\
\hline & $662(18)$ & $670(21)$ & $\mathrm{b}_{2}$ & $75(1)$ & $75(1)$ \\
\hline & $1849(1561)$ & 1797(1117) & & $98(0)$ & $95(1)$ \\
\hline & $1936(324)$ & 1841(369) & & $106(2)$ & $103(2)$ \\
\hline & $2122(372)$ & 2028(299) & & 204(1) & 213(1) \\
\hline \multirow[t]{10}{*}{$a_{2}$} & $23(0)$ & $22(0)$ & & $314(45)$ & $341(67)$ \\
\hline & $75(0)$ & $75(0)$ & & $395(32)$ & $401(26)$ \\
\hline & $105(0)$ & $106(0)$ & & $428(27)$ & $444(3)$ \\
\hline & 194(0) & $221(0)$ & & $532(35)$ & $526(17)$ \\
\hline & $269(0)$ & $270(0)$ & & $578(150)$ & $578(133)$ \\
\hline & $369(0)$ & $364(0)$ & & $660(32)$ & $669(25)$ \\
\hline & $420(0)$ & $429(0)$ & & $1831(481)$ & $1784(287)$ \\
\hline & $481(0)$ & $478(0)$ & & $2084(2354)$ & 1994(2047) \\
\hline & $590(0)$ & $586(0)$ & & & \\
\hline & $2067(0)$ & $1976(0)$ & & & \\
\hline
\end{tabular}


Table S15. Harmonic vibrational frequencies (in $\mathrm{cm}^{-1}$ ) and infrared intensities (in parentheses, in $\mathrm{km} / \mathrm{mol}$ ) for the structure IIIf-t of $\mathrm{Cr}_{2}(\mathrm{CO})_{6}(\mathrm{NO})_{2}$ with $C_{S}$ symmetry

\begin{tabular}{|c|c|c|c|c|c|}
\hline & B3LYP & BP86 & & B3LYP & BP86 \\
\hline \multirow[t]{25}{*}{$\mathrm{a}^{\prime}$} & $38(0)$ & $38(0)$ & a" & $-22(0)$ & $-8(0)$ \\
\hline & $68(0)$ & $67(0)$ & & $13(0)$ & $28(0)$ \\
\hline & $76(1)$ & $74(0)$ & & $45(3)$ & $61(0)$ \\
\hline & $83(0)$ & $82(0)$ & & $71(0)$ & $70(0)$ \\
\hline & $95(2)$ & $95(1)$ & & $75(1)$ & $75(1)$ \\
\hline & 101(1) & $97(1)$ & & $114(0)$ & $112(0)$ \\
\hline & $106(1)$ & $106(0)$ & & $277(1)$ & $278(1)$ \\
\hline & $211(0)$ & $224(0)$ & & $278(8)$ & $316(0)$ \\
\hline & $245(2)$ & $246(5)$ & & $331(6)$ & $357(0)$ \\
\hline & $348(30)$ & $362(2)$ & & $355(0)$ & $361(0)$ \\
\hline & $366(31)$ & $397(4)$ & & $368(24)$ & $377(4)$ \\
\hline & $389(38)$ & 401(29) & & $384(8)$ & $398(9)$ \\
\hline & 411(12) & $426(7)$ & & $402(3)$ & $416(0)$ \\
\hline & $436(34)$ & $444(9)$ & & $414(0)$ & $424(4)$ \\
\hline & $444(0)$ & $454(15)$ & & $466(1)$ & $468(1)$ \\
\hline & $449(12)$ & $469(6)$ & & $515(1)$ & $515(2)$ \\
\hline & $501(81)$ & $502(7)$ & & $567(0)$ & $570(1)$ \\
\hline & $512(45)$ & $532(169)$ & & $602(39)$ & $593(38)$ \\
\hline & $546(39)$ & $552(56)$ & & $1571(628)$ & $1547(525)$ \\
\hline & $566(3)$ & $576(9)$ & & $2064(595)$ & 1973(1231) \\
\hline & $604(14)$ & $605(15)$ & & $2079(1148)$ & 1971(387) \\
\hline & $641(75)$ & $640(46)$ & & $2088(2835)$ & 1981(892) \\
\hline & 1582(129) & $1564(50)$ & & $2127(120)$ & $2029(57)$ \\
\hline & $2030(558)$ & 1944(312) & & & \\
\hline & 2064(1923) & 1973(1231) & & & \\
\hline
\end{tabular}


Table S16. Harmonic vibrational frequencies (in $\mathrm{cm}^{-1}$ ) and infrared intensities (in parentheses, in $\mathrm{km} / \mathrm{mol}$ ) for the structure IIIg-t of $\mathrm{Cr}_{2}(\mathrm{CO})_{6}(\mathrm{NO})_{2}$ with $C_{2 v}$ symmetry

\begin{tabular}{|c|c|c|c|c|c|}
\hline & B3LYP & BP86 & & B3LYP & BP86 \\
\hline \multirow[t]{15}{*}{$a_{1}$} & $66(0)$ & $65(0)$ & $\mathrm{b}_{1}$ & $16(0)$ & $15(0)$ \\
\hline & $75(2)$ & $73(1)$ & & $73(1)$ & $68(1)$ \\
\hline & $117(0)$ & $109(0)$ & & $86(0)$ & $84(0)$ \\
\hline & $131(0)$ & $120(0)$ & & $352(1)$ & $350(1)$ \\
\hline & $137(0)$ & $135(0)$ & & $371(0)$ & $373(0)$ \\
\hline & $365(13)$ & $372(2)$ & & $446(30)$ & $457(9)$ \\
\hline & $397(14)$ & 409(15) & & $509(23)$ & 509(17) \\
\hline & $434(1)$ & $442(0)$ & & $638(40)$ & $628(39)$ \\
\hline & $484(2)$ & $480(2)$ & & $2038(2188)$ & 1948(1818) \\
\hline & $555(1)$ & $541(1)$ & $b_{2}$ & $46(0)$ & $22 i$ \\
\hline & 634(31) & $625(32)$ & & $79(1)$ & $75 i$ \\
\hline & $680(47)$ & $660(23)$ & & 101(1) & $80(2)$ \\
\hline & 1867(1686) & $1806(1307)$ & & $115 i$ & $93(0)$ \\
\hline & $2068(745)$ & $1975(574)$ & & $328(28)$ & $353(2)$ \\
\hline & $2122(482)$ & 2028(379) & & $379(11)$ & $381(60)$ \\
\hline \multirow[t]{10}{*}{$\mathrm{a}_{2}$} & $14 i$ & $21 i$ & & $396(3)$ & $404(6)$ \\
\hline & $25 i$ & $28 i$ & & $455(15)$ & $458(0)$ \\
\hline & $67(0)$ & $54(0)$ & & $519(89)$ & $531(0)$ \\
\hline & $86(0)$ & $84(0)$ & & $597(159)$ & $595(35)$ \\
\hline & $351(0)$ & $351(0)$ & & $666(53)$ & 644(18) \\
\hline & $363(0)$ & $362(0)$ & & 1780(119) & 1749(33) \\
\hline & $440(0)$ & $451(0)$ & & 2032(1015) & 1951(162) \\
\hline & $508(0)$ & $509(0)$ & & $2056(3617)$ & $1969(3502)$ \\
\hline & $590(0)$ & $599(0)$ & & & \\
\hline & $2033(0)$ & $1943(0)$ & & & \\
\hline
\end{tabular}


Table S17. Harmonic vibrational frequencies (in $\mathrm{cm}^{-1}$ ) and infrared intensities (in parentheses, in $\mathrm{km} / \mathrm{mol}$ ) for the structure IVa-s of $\mathrm{Cr}_{2}(\mathrm{CO})_{5}(\mathrm{NO})_{2}$ with $C_{i}$ symmetry

\begin{tabular}{|c|c|c|c|c|c|}
\hline & B3LYP & BP86 & & B3LYP & BP86 \\
\hline \multirow[t]{25}{*}{$\mathrm{a}^{\prime}$} & $33(1)$ & $32(0)$ & a" & $28(0)$ & $28(0)$ \\
\hline & $65(1)$ & $67(1)$ & & $46(0)$ & $48(0)$ \\
\hline & $72(0)$ & $71(0)$ & & $78(0)$ & $77(0)$ \\
\hline & $88(1)$ & $87(1)$ & & $95(1)$ & $96(1)$ \\
\hline & $93(0)$ & $92(0)$ & & $104(0)$ & $100(0)$ \\
\hline & $153(5)$ & $138(6)$ & & $153(2)$ & 141(1) \\
\hline & 211(1) & 193(1) & & $326(2)$ & $332(1)$ \\
\hline & $237(1)$ & $233(3)$ & & $362(0)$ & $358(0)$ \\
\hline & $348(8)$ & $330(1)$ & & $390(2)$ & $390(0)$ \\
\hline & $373(18)$ & $398(5)$ & & $396(0)$ & $384(3)$ \\
\hline & 403(11) & $409(16)$ & & $425(8)$ & $434(0)$ \\
\hline & $425(16)$ & $436(2)$ & & $437(29)$ & $454(20)$ \\
\hline & $437(18)$ & $464(1)$ & & $486(6)$ & $503(4)$ \\
\hline & $475(17)$ & $474(18)$ & & $562(24)$ & $559(19)$ \\
\hline & $509(5)$ & $512(1)$ & & $609(47)$ & $613(37)$ \\
\hline & $528(25)$ & $537(6)$ & & $2054(495)$ & $1952(222)$ \\
\hline & $600(7)$ & $600(6)$ & & 2066(1882) & 1961(1913) \\
\hline & $634(77)$ & $627(87)$ & & & \\
\hline & $697(29)$ & $680(37)$ & & & \\
\hline & 781(89) & 733(109) & & & \\
\hline & $1476(435)$ & $1501(400)$ & & & \\
\hline & 1568(317) & $1570(237)$ & & & \\
\hline & $2062(577)$ & $1958(434)$ & & & \\
\hline & $2092(2402)$ & 1994(1952) & & & \\
\hline & $2127(6)$ & $2026(61)$ & & & \\
\hline
\end{tabular}


Table S18. Harmonic vibrational frequencies (in $\mathrm{cm}^{-1}$ ) and infrared intensities (in parentheses, in $\mathrm{km} / \mathrm{mol}$ ) for the structure $\mathbf{I V b}-\mathbf{s}$ of $\mathrm{Cr}_{2}(\mathrm{CO})_{5}(\mathrm{NO})_{2}$ with $C_{1}$ symmetry

\begin{tabular}{|c|c|c|c|c|c|}
\hline & B3LYP & BP86 & & B3LYP & BP86 \\
\hline \multirow[t]{21}{*}{$\mathrm{a}$} & $29(1)$ & $30(1)$ & $\mathrm{a}$ & $445(9)$ & $464(1)$ \\
\hline & $31(1)$ & $33(0)$ & & $454(3)$ & $475(1)$ \\
\hline & $49(0)$ & $48(0)$ & & $477(5)$ & $486(4)$ \\
\hline & $59(0)$ & $53(0)$ & & $489(8)$ & 491(2) \\
\hline & $64(1)$ & $68(0)$ & & $510(17)$ & $513(22)$ \\
\hline & $76(0)$ & $76(0)$ & & $519(1)$ & $523(0)$ \\
\hline & $84(1)$ & $79(0)$ & & $570(90)$ & $557(37)$ \\
\hline & $86(1)$ & $86(1)$ & & $588(2)$ & $571(20)$ \\
\hline & $86(1)$ & $90(0)$ & & $615(29)$ & $600(38)$ \\
\hline & $96(0)$ & $97(0)$ & & $643(26)$ & $634(34)$ \\
\hline & $99(1)$ & $98(0)$ & & $666(39)$ & $646(17)$ \\
\hline & $103(2)$ & $107(2)$ & & $679(128)$ & $666(95)$ \\
\hline & 151(1) & 164(1) & & $692(15)$ & $667(27)$ \\
\hline & $312(10)$ & $297(2)$ & & $712(52)$ & $692(39)$ \\
\hline & $340(1)$ & $349(1)$ & & $1702(504)$ & $1640(404)$ \\
\hline & $379(0)$ & $387(5)$ & & $1832(2505)$ & $1774(1985)$ \\
\hline & $383(11)$ & $390(4)$ & & $1849(553)$ & $1788(385)$ \\
\hline & 394(3) & $396(4)$ & & $2016(828)$ & $1905(621)$ \\
\hline & $409(27)$ & $404(2)$ & & $2050(414)$ & 1958(439) \\
\hline & $420(2)$ & $421(10)$ & & $2065(956)$ & 1972(770) \\
\hline & $436(0)$ & $430(1)$ & & $2116(810)$ & 2022(699) \\
\hline
\end{tabular}


Table S19. Harmonic vibrational frequencies (in $\mathrm{cm}^{-1}$ ) and infrared intensities (in parentheses, in $\mathrm{km} / \mathrm{mol}$ ) for the structure IVc-s of $\mathrm{Cr}_{2}(\mathrm{CO})_{5}(\mathrm{NO})_{2}$ with $C_{1}$ symmetry

\begin{tabular}{|c|c|c|c|c|c|}
\hline & B3LYP & BP86 & & B3LYP & BP86 \\
\hline \multirow[t]{21}{*}{ A } & $5 i$ & $9(0)$ & $\mathrm{a}$ & $439(8)$ & $433(1)$ \\
\hline & $31(0)$ & $33(0)$ & & $447(3)$ & $457(2)$ \\
\hline & $54(0)$ & $53(0)$ & & $451(46)$ & $461(16)$ \\
\hline & $64(0)$ & $62(0)$ & & $468(4)$ & $468(2)$ \\
\hline & $67(0)$ & $69(0)$ & & $473(1)$ & $489(7)$ \\
\hline & $77(0)$ & $72(0)$ & & $512(7)$ & 498(1) \\
\hline & $84(0)$ & $82(0)$ & & $520(0)$ & 498(4) \\
\hline & $86(0)$ & $83(0)$ & & $557(3)$ & $544(1)$ \\
\hline & $88(1)$ & $85(1)$ & & $624(27)$ & $615(36)$ \\
\hline & $88(0)$ & $86(0)$ & & $626(37)$ & $616(53)$ \\
\hline & $98(1)$ & $96(1)$ & & $629(37)$ & $622(20)$ \\
\hline & $120(0)$ & $121(0)$ & & $650(28)$ & $632(25)$ \\
\hline & $139(0)$ & $133(0)$ & & $652(66)$ & $636(20)$ \\
\hline & $208(12)$ & $221(2)$ & & $708(7)$ & $680(3)$ \\
\hline & $327(7)$ & $328(5)$ & & $1758(405)$ & $1724(265)$ \\
\hline & $353(0)$ & $347(0)$ & & $1843(1440)$ & $1777(1187)$ \\
\hline & $371(13)$ & $375(0)$ & & $1979(557)$ & $1910(390)$ \\
\hline & $372(0)$ & $377(0)$ & & 2039(116) & $1942(277)$ \\
\hline & $393(0)$ & $385(7)$ & & $2065(2444)$ & $1977(1844)$ \\
\hline & 403(3) & $403(2)$ & & $2079(1080)$ & $1983(1015)$ \\
\hline & 414(9) & $405(10)$ & & 2123(179) & $2030(177)$ \\
\hline
\end{tabular}


Table S20. Harmonic vibrational frequencies (in $\mathrm{cm}^{-1}$ ) and infrared intensities (in parentheses, in $\mathrm{km} / \mathrm{mol}$ ) for the structure $\mathbf{I V d - t}$ of $\mathrm{Cr}_{2}(\mathrm{CO})_{5}(\mathrm{NO})_{2}$ with $C_{s}$ symmetry

\begin{tabular}{|c|c|c|c|c|c|}
\hline & B3LYP & BP86 & & B3LYP & BP86 \\
\hline \multirow[t]{25}{*}{$a^{\prime}$} & $46(1)$ & $50(0)$ & $\mathrm{al}^{\prime \prime}$ & $22(0)$ & $14(0)$ \\
\hline & $58(0)$ & $62(0)$ & & $34(0)$ & $38(0)$ \\
\hline & $72(0)$ & $71(0)$ & & $52(0)$ & $53(0)$ \\
\hline & $97(0)$ & $96(0)$ & & $73(1)$ & $80(1)$ \\
\hline & $113(0)$ & $113(0)$ & & $95(0)$ & $95(0)$ \\
\hline & $195(4)$ & $212(1)$ & & $153(0)$ & $147(0)$ \\
\hline & $245(3)$ & 253(0) & & $266(2)$ & $272(0)$ \\
\hline & $268(4)$ & $257(2)$ & & $307(2)$ & $303(0)$ \\
\hline & $296(10)$ & $310(3)$ & & $339(6)$ & $348(1)$ \\
\hline & $325(16)$ & 361(1) & & 376(9) & $396(0)$ \\
\hline & $355(36)$ & $376(31)$ & & $387(17)$ & $423(24)$ \\
\hline & $388(23)$ & $407(18)$ & & $434(0)$ & $443(0)$ \\
\hline & $407(4)$ & $435(5)$ & & $437(0)$ & $485(3)$ \\
\hline & $443(0)$ & $483(20)$ & & $485(32)$ & $515(26)$ \\
\hline & $459(5)$ & $462(4)$ & & $612(22)$ & $617(21)$ \\
\hline & $504(3)$ & $512(2)$ & & $2036(1071)$ & 1941(798) \\
\hline & $544(6)$ & $559(7)$ & & $2105(1359)$ & $1980(1441)$ \\
\hline & $566(8)$ & $621(20)$ & & & \\
\hline & $613(29)$ & $637(62)$ & & & \\
\hline & $654(67)$ & $657(39)$ & & & \\
\hline & $1470(253)$ & $1470(237)$ & & & \\
\hline & 1559(349) & $1563(325)$ & & & \\
\hline & $2053(592)$ & $1959(487)$ & & & \\
\hline & $2090(2003)$ & 1994(1854) & & & \\
\hline & $2135(211)$ & $2026(20)$ & & & \\
\hline
\end{tabular}


Table S21. Harmonic vibrational frequencies (in $\mathrm{cm}^{-1}$ ) and infrared intensities (in parentheses, in $\mathrm{km} / \mathrm{mol}$ ) for the structure IVe-t of $\mathrm{Cr}_{2}(\mathrm{CO})_{5}(\mathrm{NO})_{2}$ with $C_{1}$ symmetry

\begin{tabular}{|c|c|c|c|c|c|}
\hline & B3LYP & BP86 & & B3LYP & BP86 \\
\hline \multirow[t]{21}{*}{$\mathrm{a}$} & $19(1)$ & $13(0)$ & $\mathrm{a}$ & $390(37)$ & $388(2)$ \\
\hline & $22(0)$ & $21(0)$ & & $394(3)$ & $408(4)$ \\
\hline & $38(0)$ & $29(1)$ & & $426(34)$ & $417(1)$ \\
\hline & $43(2)$ & $44(0)$ & & $430(38)$ & $439(1)$ \\
\hline & $48(3)$ & $58(0)$ & & $436(6)$ & $451(2)$ \\
\hline & $59(1)$ & $72(1)$ & & $464(37)$ & $469(8)$ \\
\hline & $63(0)$ & $77(0)$ & & $465(18)$ & $494(36)$ \\
\hline & $70(1)$ & $83(0)$ & & $496(88)$ & $519(1)$ \\
\hline & $72(2)$ & $94(0)$ & & $499(0)$ & $536(0)$ \\
\hline & $76(1)$ & $95(1)$ & & 511(99) & $545(42)$ \\
\hline & $78(0)$ & $98(0)$ & & $538(12)$ & $608(4)$ \\
\hline & $89(3)$ & $155(0)$ & & $605(44)$ & $634(185)$ \\
\hline & $91(0)$ & 181(1) & & $641(71)$ & $650(84)$ \\
\hline & $160(0)$ & $198(2)$ & & $699(28)$ & $683(47)$ \\
\hline & $297(0)$ & $286(0)$ & & $1780(1436)$ & 1747(793) \\
\hline & $313(1)$ & $335(3)$ & & $1821(947)$ & 1794(990) \\
\hline & $330(21)$ & $348(6)$ & & $2014(1703)$ & $1831(628)$ \\
\hline & $342(1)$ & $351(0)$ & & $2028(48)$ & $1850(431)$ \\
\hline & $359(0)$ & $369(1)$ & & $2068(952)$ & 1982( 637) \\
\hline & $366(2)$ & $380(4)$ & & $2076(1278)$ & $2003(1622)$ \\
\hline & $369(16)$ & $386(1)$ & & $2120(243)$ & $2029(490)$ \\
\hline
\end{tabular}


Table S22. Harmonic vibrational frequencies (in $\mathrm{cm}^{-1}$ ) and infrared intensities (in parentheses, in $\mathrm{km} / \mathrm{mol}$ ) for the structure IVf-t of $\mathrm{Cr}_{2}(\mathrm{CO})_{5}(\mathrm{NO})_{2}$ with $C_{1}$ symmetry

\begin{tabular}{ccccc}
\hline & B3LYP & BP86 & B3LYP & BP86 \\
\hline a $19(0)$ & $22(0)$ & a & $402(20)$ & $407(2)$ \\
$32(0)$ & $35(0)$ & & $406(6)$ & $416(2)$ \\
$35(0)$ & $43(0)$ & $412(5)$ & $420(10)$ \\
$63(0)$ & $60(0)$ & & $425(2)$ & $446(1)$ \\
$65(0)$ & $69(1)$ & $436(7)$ & $451(2)$ \\
$67(1)$ & $73(0)$ & $441(13)$ & $465(5)$ \\
$70(1)$ & $73(0)$ & $460(22)$ & $489(15)$ \\
$84(1)$ & $85(0)$ & $525(57)$ & $544(67)$ \\
$86(0)$ & $87(0)$ & $547(26)$ & $554(17)$ \\
$87(0)$ & $88(0)$ & $559(4)$ & $567(26)$ \\
$113(0)$ & $120(0)$ & $616(6)$ & $617(14)$ \\
$123(0)$ & $128(0)$ & $623(9)$ & $621(10)$ \\
$126(2)$ & $140(0)$ & $625(26)$ & $629(18)$ \\
$194(3)$ & $204(0)$ & $705(10)$ & $687(4)$ \\
$307(12)$ & $314(0)$ & $1722(792)$ & $1693(644)$ \\
$318(0)$ & $323(0)$ & $1841(1040)$ & $1789(780)$ \\
$330(0)$ & $332(0)$ & $1993(1251)$ & $1907(929)$ \\
$346(15)$ & $351(5)$ & $2017(150)$ & $1925(65)$ \\
$369(1)$ & $374(0)$ & $2056(1079)$ & $1958(850)$ \\
$373(7)$ & $398(11)$ & $2078(2167)$ & $1978(1585)$ \\
$381(6)$ & $406(2)$ & $2113(279)$ & $2015(363)$ \\
\hline
\end{tabular}


Table S23. Harmonic vibrational frequencies (in $\mathrm{cm}^{-1}$ ) and infrared intensities (in parentheses, in $\mathrm{km} / \mathrm{mol}$ ) for the structure $\mathbf{V a}$ of $\mathrm{Cr}(\mathrm{CO})_{3}(\mathrm{NO})_{2}$ with $C_{2 v}$ symmetry

\begin{tabular}{|c|c|c|c|c|c|}
\hline & B3LYP & BP86 & & B3LYP & BP86 \\
\hline \multirow[t]{10}{*}{$a_{1}$} & $65(0)$ & $66(0)$ & \multirow[t]{7}{*}{$b_{1}$} & $52(0)$ & $51(0)$ \\
\hline & $89(1)$ & $85(0)$ & & $91(0)$ & $88(0)$ \\
\hline & $371(1)$ & $385(3)$ & & $339(0)$ & $330(0)$ \\
\hline & $394(14)$ & $415(7)$ & & $383(7)$ & $391(0)$ \\
\hline & $500(4)$ & $490(2)$ & & $516(30)$ & $505(27)$ \\
\hline & $561(0)$ & $546(0)$ & & $700(57)$ & $688(54)$ \\
\hline & $716(110)$ & $697(98)$ & & $1821(1541)$ & 1774(1191) \\
\hline & 1851(903) & 1797(749) & \multirow[t]{7}{*}{$\mathrm{b}_{2}$} & $85(0)$ & $82(0)$ \\
\hline & $2083(622)$ & $1985(540)$ & & $97(1)$ & $91(1)$ \\
\hline & $2145(129)$ & 2053(113) & & $345(0)$ & $345(0)$ \\
\hline \multirow[t]{4}{*}{$a_{2}$} & $88(0)$ & $83(0)$ & & $435(30)$ & $452(15)$ \\
\hline & $351(0)$ & $348(0)$ & & $518(7)$ & $506(2)$ \\
\hline & $528(0)$ & $507(0)$ & & $699(105)$ & 683(104) \\
\hline & & & & 2091(1598) & $1998(1426)$ \\
\hline
\end{tabular}

Table S24. Harmonic vibrational frequencies (in $\mathrm{cm}^{-1}$ ) and infrared intensities (in parentheses, in $\mathrm{km} / \mathrm{mol}$ ) for the structure $\mathbf{V b}$ of $\mathrm{Cr}(\mathrm{CO})_{3}(\mathrm{NO})_{2}$ with $\mathrm{C}_{2 \mathrm{v}}$ symmetry

\begin{tabular}{cccccc}
\hline & B3LYP & BP86 & & B3LYP & BP86 \\
\hline $\mathrm{a}_{1}$ & $58(0)$ & $38(1)$ & $\mathrm{b}_{1}$ & $87(0)$ & $81(0)$ \\
& $99(0)$ & $94(0)$ & & $101(0)$ & $98(0)$ \\
& $365(8)$ & $317(1)$ & & $361(0)$ & $360(0)$ \\
& $407(2)$ & $404(0)$ & & $493(3)$ & $496(0)$ \\
& $481(11)$ & $451(7)$ & & $507(30)$ & $499(42)$ \\
$504(4)$ & $488(0)$ & & $614(82)$ & $605(64)$ \\
& $713(73)$ & $676(71)$ & & $1834(2087)$ & $1786(1550)$ \\
& $1871(3)$ & $1817(0)$ & $\mathrm{b}_{2}$ & $93(0)$ & $36(1)$ \\
& $2066(1562)$ & $1974(1263)$ & & $149(0)$ & $94(0)$ \\
$\mathrm{a}_{2}$ & $2126(0)$ & $2037(0)$ & & $209 i$ & $314(1)$ \\
& $84(0)$ & $81(0)$ & & $361(0)$ & $350(0)$ \\
& $375(0)$ & $360(0)$ & & $449(8)$ & $450(7)$ \\
& $529(0)$ & $497(0)$ & & $657(85)$ & $675(71)$ \\
& & & $2054(1266)$ & $1973(1257)$ \\
\hline
\end{tabular}


Table S25. Harmonic vibrational frequencies (in $\mathrm{cm}^{-1}$ ) and infrared intensities (in parentheses, in $\mathrm{km} / \mathrm{mol}$ ) for the structure $\mathrm{Vc}$ of $\mathrm{Cr}(\mathrm{CO})_{2}(\mathrm{NO})_{2}$ with $\mathrm{C}_{2 v}$ Symmetry.

\begin{tabular}{|c|c|c|c|c|c|}
\hline & B3LYP & BP86 & & B3LYP & BP86 \\
\hline \multirow[t]{8}{*}{$a_{1}$} & $57(0)$ & $53(0)$ & $\mathrm{b}_{1}$ & $92(3)$ & $87(2)$ \\
\hline & $98(1)$ & $95(1)$ & & $317(3)$ & $312(1)$ \\
\hline & $359(5)$ & $378(4)$ & & $401(45)$ & $406(27)$ \\
\hline & $463(3)$ & $457(1)$ & & $658(26)$ & $646(25)$ \\
\hline & $640(0)$ & $616(0)$ & & $2078(1824)$ & $1976(1600)$ \\
\hline & $692(0)$ & $652(6)$ & $b_{2}$ & $70(0)$ & $63(0)$ \\
\hline & $1824(774)$ & $1767(614)$ & & $327(2)$ & $319(1)$ \\
\hline & 2131(108) & $2030(114)$ & & 477(9) & $456(4)$ \\
\hline \multirow[t]{3}{*}{$\mathrm{a}_{2}$} & $85(0)$ & $80(0)$ & & 681(1) & $672(2)$ \\
\hline & $337(0)$ & $329(0)$ & & $1787(1313)$ & 1739(998) \\
\hline & $532(0)$ & $517(0)$ & & & \\
\hline
\end{tabular}

Table S26. Harmonic vibrational frequencies (in $\mathrm{cm}^{-1}$ ) and infrared intensities (in parentheses, in $\mathrm{km} / \mathrm{mol}$ ) for the structure $\mathrm{Vd}$ of $\mathrm{Cr}(\mathrm{CO})_{4}(\mathrm{NO})$ with $C_{4 v}$ symmetry

\begin{tabular}{|c|c|c|c|c|c|}
\hline & B3LYP & BP86 & & B3LYP & BP86 \\
\hline \multirow[t]{6}{*}{$\mathrm{a}_{1}$} & $92(0)$ & $88(0)$ & $\mathrm{b}_{1}$ & $85(0)$ & $82(0)$ \\
\hline & $369(3)$ & 391(1) & & $522(0)$ & $510(0)$ \\
\hline & $498(40)$ & $497(31)$ & & $35(0)$ & $43(0)$ \\
\hline & $659(40)$ & $674(42)$ & & $339(0)$ & $356(0)$ \\
\hline & $1837(1122)$ & $1792(881)$ & & $379(0)$ & $398(0)$ \\
\hline & $2139(85)$ & $2052(69)$ & & $2066(0)$ & $1979(0)$ \\
\hline$a_{2}$ & $345(0)$ & $350(0)$ & & & \\
\hline \multirow[t]{7}{*}{$\mathrm{e}$} & $77(0)$ & $74(0)$ & & & \\
\hline & $92(1)$ & $88(1)$ & & & \\
\hline & $339(0)$ & $337(0)$ & & & \\
\hline & $425(21)$ & $433(10)$ & & & \\
\hline & $512(0)$ & $504(1)$ & & & \\
\hline & $622(95)$ & $629(87)$ & & & \\
\hline & $2062(1791)$ & $1972(1558)$ & & & \\
\hline
\end{tabular}

